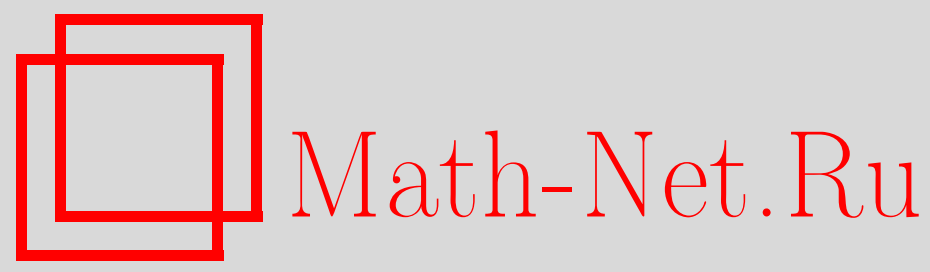

А. И. Созутов, А. К. Шлепкин, О некоторых группах с конечной инволюцией, насыщенных конечными простыми подгруппами, Матем. заметки, 2002, том 72, выпуск 3, 433-447

DOI: https://doi.org/10.4213/mzm434

Использование Общероссийского математического портала Math-Net.Ru подразумевает, что вы прочитали и согласны с пользовательским соглашением http://www . mathnet.ru/rus/agreement

Параметры загрузки:

IP: 44.207 .124 .84

26 апреля 2023 г., 18:07:20

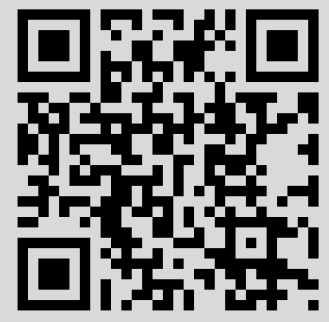




\title{
О НЕКОТОРЫХ ГРУППАХ С КОНЕЧНОЙ ИНВОЛЮЦИЕЙ, НАСЫЩЕННЫХ КОНЕЧНЫМИ ПРОСТЫМИ ПОДГРУППАМИ
}

\author{
А. И. Созутов, А.К. Шлепкин
}

В работе на основе введенного в [1] понятия насыщенности дается характеризация локально конечных простых групп $R e(P), S z(Q)$ и $L_{2}(P)$ с абелевой силовской 2-подгруппой в классах смешанных и периодических групп. Часть основных резултатов была анонсирована в [2]. Теорема 2 работы обобщает результаты статей [3], [4].

Библиография: 26 названий.

1. Формулировки основных результатов, вспомогательные утверждения. Напомним некоторые определения. Пусть $\mathfrak{X}$ - некоторое множество групा. Всякую группу $L$, изоморфную некоторой групше из $\mathfrak{X}$, будем назьвать $\mathfrak{X}$-әруппой. Группа $G$ насыщена группами из $\mathfrak{X}$, если любая ее конечная подгруппа $K$ содержится в некоторой $\mathfrak{X}$-групе $L \leqslant G$, при этом $\mathfrak{X}$ для $G$ назьвается насыщающим множеством. Множество всех конечных подгрупп $G$, изоморфных группам из $\mathfrak{X}$, будем обозначать через $\mathfrak{F}$.

Собственная подгруппа $H$ групшы $G$ назьвается сильно вложенной, если $2 \in \pi(H)$ и $2 \notin \pi\left(H \cap H^{g}\right)$ для любого элемента $g \in G \backslash H$. Инволюция $i$ групшы $G$ назьвается конечной, если для всех $g \in G$ порядки элементов $i i^{g}$ конечны [5]. Подгруппа $H$ группы $G$ назьвается сильно изолированной, если для любого неединичного элемента $h \in H$ выполняется $C_{G}(h) \leqslant H$, и бесконечно изолированной, если $C_{G}(h) \leqslant H$ для бесконечных централизаторов $C_{G}(h)$, содержащих инволюции [6].

Сформулируем основные результаты статьи.

Теорема 1. Пусть бесконечная группа $G$ с сильно вложенной подгруппой $B u$ конечной инволюиией насыщена конечными простыми подгруппами и для некоторой инволюиии $i \in G$ чентрализатор $C_{G}(i)-2$-группа. Тогда $G$ локально конечна и изоморфна одной из групп $L_{2}(Q), S z(Q)$ над подходящим локально конечным полем $Q$ характеристики 2.

Теорема 2. Если бесконечная периодическая группа $G$ содержит абелеву силовскую 2-подгруппу и насыщена конечными простыми подгруппами, то она локально конечна и изоморфна одной из групп $\operatorname{Re}(P), L_{2}(Q)$ над подходящими локально конечными полями $P, Q$.

Для доказательства этих теорем нам понадобятся следуюшие известные результаты.

Работа выполнена при поддержке Российского фонда фундаментальных исследований, грант № 99-01-00542, и Красноярского краевого фонда науки, грант № 9F0132. 
Лемма 1.1 [3, теорема 1]. Пусть периодическая группа $G$ насыщена конечными простыми неабелевыми подгруппами и некоторая силовская 2-подгруппа $S$ из $G$ является конечной группой диәдра. Тогда $G$ изоморфна локально конечной простой әруппе $L_{2}(P)$, әде $P$ - локально конечное поле нечетной характеристики.

Непосредственным следствием теоремы 3.1 из [7] является

ЛЕмма 1.2. Пусть локально конечная группа $G$ является оббединением чепоч$\kappa и G_{1}<G_{2}<G_{3}<\cdots$ конечных простых групп Шевалле лиевского ранга 1 . Тогда $G$ - простая группа Шевалле лиевского ранга 1 над локально конечным полем.

ЛЕмма 1.3. Если периодическая группа $G$ обладает конечной силовской 2-подәруппой, то все силовские 2-подгруппы группь $G$ конечны и сопряжены [8].

ЛЕмма 1.4. 2-группа с единственной инволючией либо локально ииклическая, либо (обобщенная) группа кватернионов (конечная или бесконечная) [9].

ЛЕмма 1.5. Пусть $i$ - почти регулярная конечная инволющия группь $G$. Тогда

1. $G$ - локально конечная группа;

2. $[i, G]$ содержится в $F C$-радикале группь $G$;

3. коммутант FC-радикала группь $G$ конечен [10]; в частности, периодическая группа с почти регулярной инволючией локально конечна и почти разрешима [9].

Из теоремы Горенстейна-Уолтера $[12$, с. 27] и характеризаций конечных простых неабелевых групп силовскими 2-подгруппами [13, с. 6-7] вытекает

Лемма 1.6. Пусть $G$ - конечная простая группа с абелевой силовской 2-подгруппой. Тогда $G$ изоморфна одной из групn $J_{1}, L_{2}\left(2^{n}\right), \operatorname{Re}\left(3^{2 n+1}\right), L_{2}(q)$, əде $q=3,5 \bmod 8$.

ЛЕмма 1.7 (теорема Бендера). Конечная простая группа G с сильно влохсеной подгруппой изоморфна одной из әрупп $L_{2}(q), S z(q), U_{3}(q)$, әде $q$ - подходящая степень числа 2 [12]. Если, дополнительно, иентрализатор некоторой инволючии из $G$ есть 2-группа, то $G$ изоморфна $L_{2}(q)$ или $S z(q)$.

Приведем описание некоторьх собственных подгрупп групп $L_{2}(q)$ [14].

ЛЕмма 1.8. Пусть $G=L_{2}(q)$, әде $q=p^{n}>3$ - нечетное число. Тогда справедливы следующие утверәдения:

1. силовская p-подгруппа $P$ группы $G$ әлементарная абелева и $B=N_{G}(P)=$ $P \lambda H$ - группа Фробениуса $c$ ядром $P$ и ииклическим неинвариантным множителем $H$ нечетного порядка $(q-1) / 2$;

2. все инволючии в $G$ сопряжены и силовская 2-подгруппа $T$ әруппы $G$ является группой диәдра;

3. если а - инволюиия из $G$, то $C_{G}(a)$ - группа диәдра;

4. если $G=C_{G}(a) X$, где $а-$ инволючия, $a X-$ собственная подгруппа, то $X$ сопря⿻ена с $B$ и $X \cap C_{G}(a)=1$;

5. G порождается любыми двумя различными иентрализаторами инволюuиü; 
6. если $q=3,5 \bmod 8$, то силовская 2-подгруппа $T$ группы $G$ әлементарная абелева порядка 4 и $N_{G}(T)$ - әруппа Фробениуса порядка 12.

Лемма 1.9. Пусть $G=L_{2}(q)$, где $q=2^{n}>2, P$ - силовская 2-nодгруппа группьи $G, B=N_{G}(P)$. Тогда

1. $P$ - әлементарная абелева группа и любые две силовские 2-подгруппь группь $G$ пересекаются тривиально, в частности, $C_{G}(a)=P$ для любой инволюичи $a \in P$;

2. $B=P \lambda H-$ әруппа Фробениуса $с$ ядром $P$ и ииклическим неинвариантным множителем $H$ порядка $q-1$, действуюшим транзитивно на множестве $P^{\#}$, в частности, все инволюиии в $G$ сопряжены;

3. $N=N_{G}(H)=H \lambda\langle v\rangle-$ zpynna $\partial и ә \partial p a ;$

4. если $K$ - подгруппа в $G$ и $K$ обладает нормальной подгруппой нечетного порядка, то $N_{G}(K)$ - группа диәдра;

5. G порожсдается любыми двумя силовскими 2-подгруппами.

Описание строения подгрупш в $S z(q)$ можно найти в [14], [15].

Лемма 1.10. Группа $S z(q)$ имеет следующие максимальные подгруппь:

1. $B=P \lambda H$ - әруппа Фробениуса порядка $q^{2}(q-1)$ с ядром $P$ порядка $q^{2}$ (силовская 2-подгруппа) и ииклическим неинвариантным множителем $H$ (подәруппа Картана) порядка $q-1$, транзитивно действующим на множествах неединичных әлементов из $Z(P)$ и $P / Z(P)$;

2. группу диэдра порядка $2(q-1)$ (нормализатор подгруппь Картана);

3. группу Фробениуса $\langle a\rangle \lambda\langle b\rangle$, где $|a|=q+r+1,|b|=4 u r^{2}=2 q$;

4. группу Фробениуса $\langle a\rangle \lambda\langle b\rangle$, где $|a|=q-r+1,|b|=4 u r^{2}=2 q$;

5. $S z(s)$, где $s^{t}=q u t-$ простой делитель числа $2 k+1 ;$ здесь $q=2^{2 k+1}$.

Из теоремы 3.33 [12] и результатов работ [16], [17] следует

Лемма 1.11. Пусть $G=\operatorname{Re}(q)$, әде $q=3^{2 n+1}>3, a-$ инволюиия из $G, T$ силовская 2-подгруппа в $G, B-$ подгруппа Бореля. Тогда

1. $|G|=q^{3}(q-1)\left(q^{3}+1\right),|B|=q^{3}(q-1)$;

2. $B=U \lambda H=N_{G}(U), H-$-иклическая группа порядка $q-1, N_{G}(H)=H \lambda\langle v\rangle-$ группа диэдра, $|O(H)|=\frac{1}{2}|H|, U \lambda O(H)$ - группа Фробениуса;

3. $T$ - әлементарная абелева группа порядка $8, C_{G}(T)=T u H=N_{G}(T)=$ $T \lambda(\langle b\rangle \lambda\langle d\rangle)$, әде $\langle b\rangle \lambda\langle d\rangle-$ әруппа Фробениуса порядка 21 ;

4. $C_{G}(a)=\langle a\rangle \times L$, əдe $L \simeq L_{2}(q)$;

5. $G=\left\langle H, C_{G}(a)\right\rangle=\left\langle S, C_{G}(a)\right\rangle$, где $S$ - произвольная силовская 2-подгруппа группь $G$, не содерэсащая инволюиии а;

6. все инволюции из $G$ сопряжены в $G$.

Из описания максимальных подгрупп в $R e(q)[17]$ вытекает

Лемма 1.12. Пусть $G=R e(q)$, где $q=3^{2 n+1}>3, U-$ силовская 3-подгруппа из $G$ и $D<G$ - некоторая подгруппа нечетного порядка. Тогда справедливо хотя бъ одно из утверждений:

1. $D \leqslant U \lambda A_{0}=O(B)$, где $B$ - подгруппа Бореля, $A_{0}=O(H)$ - ииклическая группа порядка $(q-1) / 2$; 
2. D содержится в одной из групп Фробениуса вида $A_{i} \lambda\left\langle b_{i}\right\rangle, i=1,2,3, c$ ииклическими ядрами $A_{i}$ порядков $(q+1) / 4, q+1-3^{n+1}, q+1+3^{n+1}$ соответственно и неинвариантными множителями $\left\langle b_{i}\right\rangle$ порядка 3.

При этом $C_{G}\left(A_{0}\right)$ и $C_{G}\left(A_{1}\right)$ содержст инволюиии, а $A_{2}$ и $A_{3}$ инвертируются инволюииями.

Как известно [18] (см. также [12, с. 90-93]), имеет место следующая

Лемма 1.13. Пусть $G=J_{1}$ - первая әруппа Янко, $а$ - инволюиия из $G, T-$ силовская 2-подгруппа в $G$. Тогда

1. $T$ - әлементарная абелева группа порядка $8, C_{G}(T)=T$ и $H=N_{G}(T)=$ $T \lambda(\langle b\rangle \lambda\langle d\rangle)$, где $\langle b\rangle \lambda\langle d\rangle-$ группа Фробениуса порядка 21 ;

2. $C_{G}(a)=\langle a\rangle \times L$, əдe $L \simeq L_{2}(4) \simeq L_{2}(5)$;

3. $G=\left\langle H, C_{G}(a)\right\rangle=\left\langle S, C_{G}(a)\right\rangle$, әде $S$ - произвольная силовская 2-подгруппа группь $G$, не содержащая инволюиии а;

4. все инволюиии из $G$ сопряжены в $G$.

Утверждения нижеследующих лемм можно найти в [5], [19], [20].

Лемма 1.14. Пусть группа $G$ содержит сильно вложенную подгруппу $B$ иконечную инволюиию. Тогда все инволючии в $G$ сопряжены и все инволюиии из $B$ сопряжены в $B$. В частности, любая пара инволюиий в $G$ порождает конечную noдzpynny.

Лемма 1.15. Пусть $B$ - собственная подгруппа группь $G$ с конечной инволюиией. Тогда следующие условия әквиваленть:

1. подгруппа $B$ сильно влохсена в $G$;

2. подгруппа $B$ содержит инволючию, $C_{G}(i) \leqslant B$ для каждой инволючии $i \in B$ ине все инволюиии из $G$ содержатся в $B$.

Лемма 1.16. Пусть $G$ - группа с сильно вложснной подгруппой $B$ и конечной инволюиией. Тогда

1. между множеством инволюиий из $B$ и множеством инволюций любого правого смежного класса $B g$, где $g \in G$, можсно установить взаимно однозначное соответствие, при этом если $i$ - фиксированная инволюция из $B$, то каждой инволюиии $k \in B$ (в том числе и инволюции $i$ ) соответствует единственная инволюиия $j_{k} \in B g$ такая, что $g^{-1} \mathrm{~kg}=j_{k} i j_{k}$;

2. любой әлемент $g \in G$ обладает представлением $g=h j$, где $h \in B, a j-$ некоторая инволюиия;

3. для любой инволюиии $j \in G \backslash B$ в подгруппе $B$ существует мнохество $M_{j}$ строго вещественных относительно $j$ әлементов той же мощности, что и множество инволюций из $B$.

Лемма 1.17. Пусть $G$ - әруппа с конечной инволючией и сильно вложенной подгруппой $B, i \in B$ и $j \in G \backslash B-$ произвольные инволюиии. Пусть далее $H_{j}=B \cap B^{j}$ и $T_{j}$ - подгруппа в $B$, порожденная всеми строго вещественными относительно ј әлементами из В. Тогда

1. $T_{j} \leqslant H_{j}, H_{j}-$ подгруппа без инволюиий, $\left\langle j, T_{j}\right\rangle=T_{j} \lambda\langle j\rangle u\left\langle j, H_{j}\right\rangle=H_{j} \lambda\langle j\rangle$; 
2. в каждом смежном классе $C_{B}(i) \cdot b$, әде $b \in B$, существует единственный строго вещественный әлемент относительно инволючии $j$ и его порядок конечен и нечетен;

3. $B=T_{j} \cdot C_{B}(i)$, в частности, $i^{T_{j}}$ - множество всех инволюций әруппь $B$;

4. если $b$ - неединичный строго вещественный относительно инволюции $j \in$ $G \backslash B$ әлемент из $B$, то $C_{B}(b)$ не содерэсит инволючий.

ЛЕмма 1.18 [6, теорема 2]. Пусть группа $G$ содержит конечную инволючию, сильно влохсеную бесконечно изолированную подгруппу $B$ и выполняются следующие условия:

1. чентрализатор $C_{G}(k)$ некоторой инволючии $k \in G$ не содержит бесконечных подгрупп, в которых каждый неединичный әлемент почти регулярен;

2. хотя бы одно из пересечений $B \cap B^{g}$, где $g \in G \backslash B$, бесконечно;

3. существует әлемент $b \in B$, инвертируемый некоторой инволюцией $j$ из $G$, иентрализатор $C_{G}(b)$ которого не содержится в $B$.

Тогда все инволюиии в $G$ сопряжсены, $F=\left\langle b^{G}\right\rangle$ - периодическая абелева подгруппа, инвертируемая каждой инволющией из $G, u G=C_{G}(F) C_{G}(k)$.

ЛЕмма 1.19 (Лемма Бусаркина). Пусть $G$ - әруппа с конечной инволюиией $i$. Если $C_{G}(i)=\langle i\rangle$, то $G=F \lambda\langle i\rangle$, где $F-$ периодическая абелева подгруппа из $G$, инволюиия $i$ инвертирует $F[19$, предложение 4.2].

Наконец, нам понадобится лемма, обобщающая одно предложение из [21].

Лемма 1.20. Пусть $G$ - произвольная группа $u\left\{L_{i}=F_{i} \lambda\langle a\rangle \mid i \in J\right\}$ - некоторое множество ее фробениусовых подгрупп с неинвариантным множителем $\langle a\rangle$ и ядрами $F_{i}$. Если $X=\cup F_{i} \subseteq N_{G}(X)$, то для подәрупп $B=\langle X, a\rangle$ u $T=\langle X\rangle$ справедливы следующие утверждения:

1. $B=T \lambda\langle a\rangle$ и каждый әлемент из $B \backslash T$ сопряжен в $B$ с подходящим неединичным әлементом из подгруппь $\langle a\rangle$;

2. если все подгруппь $F_{i}$, где $i \in J$, абелевы $u|a|=n<\infty$, то $T$ - нильпотентная группа;

3. если все подгруппы $F_{i}$, әде $i \in J$, абелевые периодические группы $u|a|=$ $n<\infty$, то $B$ - локально конечная группа Фробениуса.

ДокАЗАТЕЛЬСТво. 1. По условиям леммы $T \leqslant N_{G}(X)$ и для любьх элементов $x \in X$ и $r \in\langle a\rangle^{\#}$ найдется элемент $v \in X \subseteq T$ такой, что $(r x)^{v} \in\langle a\rangle^{\#}$. Пусть теперь $t=x_{1} x_{2} \ldots x_{n}$ - произвольньй элемент из $T$, где $x_{1}, \ldots, x_{n} \in X$. По индуктивному предположению мы можем считать, что $r x_{1} \ldots x_{n-1}=s^{b}$ для подходящих элементов $s \in\langle a\rangle^{\#}$ и $b \in T$. Так как $y=b x_{n} b^{-1} \in X$ по условиям леммы, то по вьшедоказанному элемент $r t=s^{b} x_{n}=s^{b} y^{b}=(s y)^{b}$ сопряжен с некоторым неединичным элементом из $\langle a\rangle$. Следовательно, каждьй элемент из $\langle a\rangle^{\#} T$ сопряжен в $T$ с неединичным элементом из $\langle a\rangle$. В частности, $\langle a\rangle \cap T=1$, так как из $1 \neq r \in\langle a\rangle \cap T$ по доказанному следует, что единица $1=r r^{-1}$ сопряжена с неединичным элементом из $\langle a\rangle$, что невозможно. Таким образом, $r^{B}=r^{T}=r T$ для любого $r \in\langle a\rangle^{\#}$; ввиду условий леммы подгруппа $T$ нормальна в $B$ и $B=T \lambda\langle a\rangle$. Утверждение доказано. 
2. Пусть $t \in T, x \in X$ - произвольные элементы и $1 \leqslant m<n$. По условиям леммы и первому утверждению $\left\langle x, t a^{m}\right\rangle^{v} \leqslant F_{i} \lambda\langle a\rangle$ для подходящих $v \in T$ и индекса $i$. Отсюда следует, что $x^{t a^{m}} \in C_{G}(x)$ и $x^{B \backslash T} \subseteq C_{G}(x)$. Из первого утверждения также получаем $\left|x^{t} a^{-1}\right|=n$ и, следовательно,

$$
1=\left(x^{t} a^{-1}\right)^{n}=x^{t} \cdot x^{t a} \cdot x^{t a^{2}} \cdots x^{t a^{n-1}},
$$

откуда заключаем, что

$$
x^{t}=\left(x^{t a} \cdot x^{t a^{2}} \cdots x^{t a^{n-1}}\right)^{-1} \in C_{G}(x) .
$$

Таким образом, $x^{B} \subseteq C_{G}(x),\left\langle x^{B}\right\rangle$ абелева подгруппа для любого $x \in X$ и по теореме Плоткина [22] подгруппа $T$ локально нильпотентна. Так как любой элемент простого порядка из $\langle a\rangle$ по первому утверждению леммы индуцирует на $T$ расщепляющий автоморфизм, то по теореме из [23] $T$ - нильпотентная группа. Утверждение доказано.

3. Обозначим $\pi=\pi(\langle a\rangle)$. Ввиду условий утверждения подгруппа $T$ порождена $\pi^{\prime}$-элементами, а по утверждению $2 T$ - нильпотентная група. Следовательно, $T-$ $\pi^{\prime}$-группа и из первого утверждения легко следует, что $C_{T}(r)=1$ для любого $r \in\langle a\rangle^{\#}$. Это означает, что $(B,\langle a\rangle)$ - пара $\Phi$ робениуса и $B$ - локально конечная группа Фробениуса с неинвариантным множителем $\langle a\rangle$ и ядром $T$. Лемма доказана.

2. Доказательство теоремы 1. На протяжении этого раздела группа $G$ и ее собственная подгрупша $B$ удовлетворяют условиям теоремы 1.

ЛЕмма 2.1. Пусть $v$ - произвольная инволюиия из $G \backslash B$. Тогда

1. подгруппа $H=B \cap B^{v}$ абелева и инволюиия $v$ инвертирует $H$;

2. подгруппа $Z$, порожденная всеми инволюииями из $B$, әлементарная абеле$\boldsymbol{в а ;}$

3. силовская 2-подгруппа $U$ группь $B$ нормальна в $B$ и либо совпадает с $Z$, либо является локально конечной группой периода 4 , причем $Z(U)=Z$;

4. $B=U \lambda H-$ локально конечная группа Фробениуса $с$ ядром $U$ и неинвариантным множителем $H$;

5. Н - локально ииклическая әруппа.

ДоКАЗАТЕЛЬСТво. Ввиду леммы 1.14 все инволюции в $G$ сопряжены и все инволюции из $B$ сопряжены в $B$. Поэтому централизатор любой инволюции из $G$ является 2 -группой и каждая инволюция в $G$ конечна.

1. В силу лемм $1.14,1.16 H-$ группа без инволюций и $C_{H}(v)=1$. Ввиду лемм $1.5,1.19$ $H$ - периодическая абелева группа, состоящая из всех строго вещественных относительно инволюции $v$ элементов из $B$. Утверждение доказано.

2. Пусть $i-$ произвольная инволюция из $B$. В силу леммы 1.14 подгрупша $D=\langle i, v\rangle$ конечна и по условиям теоремы $D$ содержится в конечной простой неабелевой подгруппе $L$ из $G$. Так как $L \not B$ и $i \in L \cap B$, подгруппа $L \cap B$ сильно вложена в $L$. Поскольку все инволюции в $G$ сопряжены, то централизатор каждой из них является 2-группой и ввиду результата Бендера (лемма 1.7) заключаем, что $L$ изоморфна одной из групп $L_{2}(Q)$, $S z(Q)$ над конечным полем $Q$ характеристики 2. При этом подгруппа $L \cap B$ содержит инволюцию $i$ и совпадает с нормализатором $N_{L}(S)$ силовской 2 -подгрупшы $S$ в $L$ 
(леммы $1.9,1.10$ ). Как вытекает из строения групп $L_{2}(Q)$ и $S z(Q)$ (леммы $\left.1.9,1.10\right)$, $N_{L}(S)=S \lambda\langle A\rangle-$ группа рробениуса с неединичным циклическим неинвариантньм множителем $A=\langle a\rangle$, инвертируемым инволюцией $v$, и $L_{i}=\langle a, i\rangle=F_{i} \lambda\langle a\rangle$ - группа Фробениуса с абелевым ядром $F_{i}$. Очевидно, что $a \in H$ и $H \leqslant C_{G}(a)$ по первому утверждению леммы. Далее, ввиду утверждения 3 леммы 1.17 множество инволюций в $B$ совпадает с множеством $i^{H}$. Таким образом, если $j$-произвольная инволюция из $B$, то $j=i^{h}$ для подходящего элемента $h \in H$ и $L_{j}=\langle a, j\rangle=\left\langle a, i^{h}\right\rangle=L_{i}^{h}$ - конечная группа Фробениуса с неивариантным множителем $\langle a\rangle$ и элементарным абелевьм ядром $F_{j}$. Пусть $J$ - множество всех инволюций из $B$. Тогда объединение $X$ всех ядер $F_{j}$, где $j \in J$, совпадает с $J$, в частности, $X \leqslant N_{G}(X)$. Применяя лемму 1.20 , приходим к выводу, что подгруппа $\langle J, a\rangle=Z \lambda\langle a\rangle$ - локально конечная группа Фробениуса с нильпотентным ядром $Z$. Отсюда следует, что $Z$ - характеристическая в $B$ нильпотентная 2-подгруппа.

Пусть $K$-произвольная подгруппа из $Z$, порож денная конечным числом инволюций. Ввиду вышедоказанного $K$ - конечная 2-группа и по условию теоремы $K<L<G$, где $L$ - конечная простая неабелева группа. Так как $K \leqslant L \cap Z$ и 2 -подгруппа $Z$ нормальна в $B$, то $L \& B$ и подгрупша $L \cap B$ сильно вложена в $L$. Как и выше, заключаем, что $L$ изоморфна $L_{2}(Q)$ или $S z(Q)$ над конечным полем $Q$ характеристики 2 , при этом $L \cap B$ совпадает с нормализатором $N_{L}(S)$ силовской 2-подгруппы $S$ в $L$. Понятно, что $K \leqslant S$ и, учитывая строение подгрупшы $S$ (леммы $1.9,1.10,[12$, с. 166$])$, приходим к выводу, что $K$ - элементарная абелева группа. Ввиду произвольности $K$ заключаем, что и подгруппа $Z$ элементарная абелева. Утверждение доказано.

3. Пусть теперь $K$ - произвольная конечная 2 -подгруппа из $B$. Снова по условиям теоремы $K<L<G$, где $L$ - конечная простая неабелева группа, $L \cap Z \neq 1$; ввиду нормальности подгрупшы $Z$ в $B$ и простоты $L$ имеем $L \& B$ и подгруппа $L \cap B$ сильно вложена в $L$. Как и выше, заключаем, что $L$ изоморфна $L_{2}(Q)$ или $S z(Q)$ над конечным полем $Q$ характеристики $2, L \cap B$ совпадает с нормализатором $N_{L}(S)$ силовской 2 -подгрупшы $S$ в $L, K \leqslant S$ и $K$ - либо элементарная абелева группа, либо группа периода 4 , причем $Z \cap S=Z(S)$. В силу утверждения 2 леммы мы можем считать, что $K \cap Z$ может быть произвольной наперед заданной подгруппой из $Z$. Отсюда вытекает включение $K \leqslant C_{B}(Z)$. Более того, для любых инволюций $z, x \in Z$ вьполняется равенство $C_{G}(z)=C_{B}(z)=C_{B}(x)=C_{G}(x)$. Это означает, что $C_{G}(Z)=C_{G}(z)=U-$ силовская 2 -подгруппа из $B$ периода $\leqslant 4$ и ввиду утверждения 2 леммы либо $U=Z$, либо $U / Z$ - элементарная абелева 2 -группа, причем $U$ нормальна в $B$ как централизатор характеристической подгруппы $Z$. Утверждение доказано.

4. Пусть $i$-произвольная инволюция из $B$. По утверждениям 2,3 леммы $C_{B}(i)=U-$ нормальная в $B$ нильпотентная силовская 2 -подгруппа группы $G$. Из утверждения 1 леммы и утверждений 3,4 леммы 1.17 легко следует, что $B=U \lambda H$ - локально конечная группа Фробениуса с ядром $U$ и неинвариантным множителем $H$.

5. Ввиду утверждений 1,4 леммы $H$ - периодическая абелева групша регулярных инволюций абелевой групшы $Z$. Как хорошо известно (см., например, [14]), из этого следует локальная цикличность подгруппы $H$. Лемма доказана.

Лемма 2.2. Пусть $v$ - произвольная инволюиия из $G \backslash B$. Тогда

1. подгруппа $H=B \cap B^{v}$ сильно изолирована в $G$;

2. множество инволюций из $G \backslash B$ совпадает с $v^{B}$; 
3. әруппа $G$ действует дважды транзитивно на множестве $\Omega$ всех своих подгрупп, сопряженных с $B$;

4. только единичный әлемент группь $G$ оставляет на месте три различные точки из $\Omega$.

ДокАЗАТЕЛЬСтво. 1. Из леммы 2.1 следует неравенство $m(U) \geqslant 2$. Покажем, что силовская 2-подгруппа $U$ из $B$ содержит бесконечно много инволюций. Предположим противное. Тогда ввиду леммы $2.1 B$-конечная подгруппа и по условиям теоремы $B<L<G$, где $L$ - конечная простая группа, изоморфная либо $L_{2}(Q)$, либо $S z(Q)$ над полем $Q$ характеристики 2 (лемма 1.7), поскольку централизатор каждой инволюции из $G$ есть 2-группа (лемма 2.1). Понятно, что подгруппа $L$ содержит централизатор $C_{G}(i)$ каждой своей инволюции и по лемме $1.15 L$ сильно вложена в $G$. Но тогда ее строение описьвается леммой 2.1, противоречие. Следовательно, множество инволюций в $B$ бесконечно. Ввиду леммы 2.1 вьполняется условие 1 леммы 1.18 , а с учетом лемм $1.16,1.17$ и условие 2 леммы 1.18. Поскольку заключение данной леммы для $G$ неверно, то для любого элемента $t \in H^{\#}$ вытекает равенство $C_{G}(t)=H$. Последнее означает, что подгруппа $H$ сильно изолирована в $G[14]$.

2. Пусть $j, k$ - произвольные различные инволюции из $G \backslash B$. Ввиду леммы 2.1 пересечения $H_{j}=B \cap B^{j}$ и $H_{k}=B \cap B^{k}$ суть дополнительные множители $\Phi$ робениуса в группе $B$ и поэтому сопряжены в $B$ с $H$. Из леммы 2.1 следует, что инволюции $j$ и $k$ инвертируют соответственно подгруппы $H_{j}$ и $H_{k}$. Из сильной изолированности подгрупп $H_{j}$ и $H_{k}$ (утверждение 1 леммы) вытекает, что все инволюции из $G \backslash B$, инвертирующие данные подгруппы, содержатся в $H_{j} \lambda\langle j\rangle$ и $H_{k} \lambda\langle k\rangle$ соответственно. Поэтому из сопряженности подгрупш $H_{j}$ и $H_{k}$ с $H$ в $B$ следует сопряженность подгрупा $H_{j} \lambda\langle j\rangle$ и $H_{k} \lambda\langle k\rangle$, причем с помошью некоторого элемента $u \in U$. Поскольку в $H_{k} \lambda\langle k\rangle$ все инволюции сопряжены ввиду нечетности подгрупшы $H$ (лемма 2.1), то $j=k^{b}$ для некоторого элемента $b \in B$.

3. Из утверждения 2 леммы следует, что $B$ действует транзитивно (сопряжениями) на множестве всех подгрупп $C_{G}(j)$, где $j$ - инволюция из $G \backslash B$. Ввиду леммы 2.1 это означает, что $B$ действует транзитивно на множестве $\Omega$ всех сопряженных с $B$ и отличных от $B$ подгрупп групшы $G$. Так как $N_{G}(B)=N_{G}(U)=B$, то $G$, очевидно, 2 -транзитивна на $\Omega$ и $B=G_{\alpha}$ - стабилизатор точки $\alpha=B \in \Omega$.

4. Ввиду лемм $1.16,1.17,1.18$ и 2.1 для инволюции $k \in N_{G}(H)$ имеет место равенство $H=B \cap B^{k}$. Поскольку $U \cap N_{G}\left(B^{k}\right)=1$ (лемма 2.1), то $H=G_{\alpha \beta}$, где $\beta=B^{k} \in \Omega$. Следовательно, подгруппа $H$ совпадает со стабилизатором $G_{\alpha \beta}$ двух точек $\alpha, \beta \in \Omega$. Заметим, что $\Omega \backslash\{\alpha\}=\beta^{B}=\beta^{H U}=\beta^{U}$. Поскольку $H \cap U=1$, то для $1 \neq x \in U$ выполняется $\beta^{x}=\gamma \neq \beta$. Если $h \in H$ и $\gamma^{h}=\gamma$, то $\beta^{x h}=\beta^{x}$ и $x h x^{-1} \in H \cap x H x^{-1}=1$. Значит, $h=1$ и $G_{\alpha \beta \gamma}=1$. Таким образом, $G_{\alpha \beta \gamma}=1$ для каждой точки $\gamma \in \Omega \backslash\{\alpha, \beta\}$. Лемма доказана.

ЛЕмма 2.3. Подгруппа В изоморфна подгруппе Бореля либо группы $L_{2}(Q)$, либо группы $S z(Q)$ над подходящим локально конечным полем $Q$ характеристики 2.

ДокАЗАТЕЛЬСТво. Покажем вначале, что $B$ - счетная группа. По лемме $2.1 B=$ $U \lambda H-$ група $\Phi$ робениуса с периодическим локально циклическим неинвариантньм множителем $H$. Понятно, что подгруппа $H$ счетна. По лемме $1.17 H$ транзитивна на множестве инволюций из $U$, которое вместе с единицей составляет центр $Z=Z(U)$ 
группы $U$. Следовательно, $Z$ - счетная группа и по лемме 4.5 [5] $Z \lambda H$ изоморфна подгрупше Бореля группы $L_{2}(Q)$ над подходящим локально конечньм полем $Q$ характеристики 2. В частности, если $U=Z$, то лемма доказана.

Пусть $U \neq Z, x, y$ - произвольные элементы порядка 4 из $U$ и $K$ - любая конечная 2 -подгруппа из $B$, содержащая элементы $x$ и $y$. По условиям теоремы $K<L<G$, где $L$ - конечная простая группа. Понятно, что $L \simeq S z(q)$ и $M=B \cap L=(M \cap U) \lambda T$-нормализатор силовской 2 -подгруппы в $L$. Пусть $Z_{1}=Z \cap M$. Как вытекает из леммы 1.10 , смежные классы $x Z_{1}$ и $y Z_{1}$ сопряжены в $B \cap L$. Отсюда выводим, что $H$ действует транзитивно на множестве смежных классов $U / Z$, отличных от $Z$. В частности, $U$ - счетная группа и $B$ также счетна. Поле $Q$, ассоциированное с подгруппой $Z \lambda H$, представим в виде объединения цепи конечных подполей:

$$
Q_{1} \subset Q_{2} \subset Q_{3} \subset \cdots, \quad Q=\cup Q_{i} .
$$

Ввиду доказанного выше изоморфизма группы $Z \lambda H$ подгруппе Бореля $L_{2}(Q)$ цепи 1 соответствуют следующие подгруппы

$$
\begin{gathered}
H_{1}<H_{2}<H_{3}<\cdots, \quad H=\cup H_{i}, \quad H_{i}^{v}=H_{i}, \\
Z_{1}<Z_{2}<Z_{3}<\cdots, \quad Z=\cup Z_{i},
\end{gathered}
$$

такие, что $Z_{k} \lambda H_{k}$ изоморфна подгруппе Бореля в $L_{2}\left(Q_{k}\right)$. Пусть $B_{k}^{\prime}-$ конечная подгруппа из $B$, содержащая подгруппу $Z_{k} \lambda H_{k}$ и некоторьй элемент порядка 4 из $U$. По условиям теоремы $B_{k}^{\prime}<L<G$, где $L$ конечна, проста и, как нетрудно убедиться, изоморфна $S z\left(Q_{k}^{\prime}\right)$ для подходящего поля $Q_{k}^{\prime}$. Поскольку $B_{k}$ содержится в подгруппе Бореля групшы $L$, то с помошью леммы 1.10 вьводим, что поле $Q_{k}^{\prime}$ содержит подполе $Q_{k}$, причем $Z_{k} \lambda H_{k}$ содержится в подгруппе Бореля $B_{k}$ группы $L_{1}<L, L_{1} \simeq S z\left(Q_{k}\right)$. Выберем подгрупшы $B_{k}$ таким образом, чтобы они составляли цепочку

$$
B_{1}<B_{2}<B_{3}<\cdots .
$$

Так как объединение $B^{*}$ этой цепочки содержит подгруппы $H, Z(U)$ и $H$ действует на множестве неединичных элементов факторгруппы $U / Z(U)$ транзитивно, то $B^{*}=B$. Наконец, стандартными рассуждениями доказьвается изоморфизм группы $B$ и подгруппы Бореля групшы $S z(Q)$ (см., например, лемму 5.6 из [26]). Лемма доказана.

ЛЕмма 2.4. G изоморфна $L_{2}(Q)$ или $S z(Q)$ над подходящим локально конечным полем $Q$ характеристики 2.

ДокАЗАТЕЛЬСТво. Лемма вытекает из лемм 2.2, 2.3 теоремы 4.1 [5] и теоремы 2 [26].

3. Доказательство теоремы 2. Пусть далее группа $G$ удовлетворяет условиям теоремы 2 .

ЛЕмма 3.1. G- простая группа. 
ДоКАЗАТЕЛЬСтво. Пусть $1 \neq H \triangleleft G, a \in H^{\#}$ и $b \in G \backslash H$. По условиям теоремы найдутся подгруппы $K$ и $M$ из $\mathfrak{F}$ такие, что $\langle a\rangle<K,\langle b\rangle<M$. Очевидно, что $K \leqslant H$ и $M \cap H=1$, в частности, в $H$ и $G \backslash H$ найдутся инволюции $i$ и $j$. В силу периодичности группы $G$ подгруппа $R=\langle i, j\rangle$ конечна и по условиям теоремы $R<L \in \mathfrak{F}$. Так как $L \cap H \neq 1, L \backslash H \neq \varnothing$ и $L$ - простая группа, получаем противоречие. Следовательно, $G$ - простая групша и лемма доказана.

ЛЕмма 3.2. Если некоторая силовская 2-подгруппа $U$ группь $G$ конечна, то она әлементарная абелева порядка 4 или 8.

ДоКАЗАТЕЛЬСТво. Пусть $|U|=2^{n}>8$. По условиям теоремы $U<L<G$, где $L-$ конечная простая группа и ввиду теоремы Уолтера (лемма 1.6) $L \simeq L_{2}\left(2^{n}\right)$. По лемме $1.9 U$ - элементарная абелева группа. Пусть $i$ - инволюция из $U$. Поскольку $G$ бесконечна и проста (лемма 3.1 ), то ввиду леммы 1.5 подгруппа $C_{1}=C_{G}(i)$ бесконечна. Предположим, что $C_{1}$ локально конечна. Тогда $U$ строго содержится в некоторой конечной подгруппе $K$ из $C_{1}$. По условиям теоремы $K<L<G$, где $L$ - конечная простая группа. По теореме Уолтера (лемма 1.6) $L \simeq L_{2}\left(2^{n}\right)$, что противоречит строению централизаторов инволюций в $L_{2}\left(2^{n}\right)$ (лемма 1.9). Следовательно, группа $C_{1}$ не локально конечна. Пусть $j \in U$ - инволюция, отличная от $i$. Ввиду леммы 1.5 подгруппа $C_{2}=C_{G}(j) \cap C_{1}$ бесконечна. Понятно, что $U<C_{2}$ и если $C_{2}$ локально конечна, то, как и вьше, приходим к противоречию. Следовательно, $C_{2}-$ не локально конечна. Но тогда для инволюции $k \in U \backslash\langle i, j\rangle$ подгруппа $C_{3}=C_{G}(k) \cap C_{2}$ бесконечна и по тем же соображениям не локально конечна. Продолжая этот процесс, получаем, что подгруппа $C_{n}$ бесконечна. Поскольку $C_{n} \leqslant C_{G}(U)$, приходим к выводу, что $C_{G}(U)$ содержит элемент нечетного порядка. Как показано выше, это невозможно. Лемма доказана.

ЛЕмма 3.3. Если порядок некоторой силовской 2-подгруппы $U$ из $G$ равен 4 , то $G$ локально конечна и изоморфна группе $L_{2}(P)$ над подходящим локально конечным полем $P$ нечетной характеристики.

ДокАЗАТЕЛЬСТво. По условиям теоремы $U<L<G$, где $L$ - конечная простая группа. Ввиду известной теоремы Бернсайда [24] подгруппа $U$ элементарная абелева и в то же время группа диэдра. По лемме $1.1 G$ изоморфна группе $L_{2}(P)$ над подходяшим локально конечным полем $P$ нечетной характеристики.

ЛЕмма 3.4. Если некоторая силовская 2-подгруппа $U$ из $G$ бесконечна, то $G$ локально конечна и изоморфна группе $L_{2}(Q)$ над подходящим локально конечным полем $Q$ характеристики 2.

ДокАЗАТЕЛЬСТво. Пусть $K$ - произвольная конечная подгрупша из $U$ порядка $>8$. По условиям теоремы $K<L<G$, где $L$ - конечная простая группа. Ввиду леммы 1.6 $L \simeq L_{2}\left(2^{n}\right)$ и по лемме $1.9 K$ - элементарная абелева группа. Следовательно, и группа $U$ элементарная абелева.

По той же теореме Уолтера насьщающее множество $\mathfrak{F}$ для грушшы $G$ может содержать только конечные простые групшы из списка $J_{1}, L_{2}\left(2^{n}\right), \operatorname{Re}\left(3^{2 n+1}\right), L_{2}(q)$, где $q=$ $3,5 \bmod 8$. Пусть $i$ - произвольная инволюция из $U$ и предположим, что $i \in L<G$, где $L-$ конечная простая группа, изоморфная $J_{1}$ или $R e\left(3^{2 n+1}\right)$. Ввиду лемм $1.11,1.13$ нормализатор силовской 2 -подгрупшы $T$ в $L$ имеет порядок 168 и содержит фробениусову подгрупу $K=\langle b\rangle \lambda\langle d\rangle$ порядка 21 . Пусть $i, j$-произвольные различные инволюции из 
$N_{G}(T) \backslash T$. Поскольку $D=\langle i, j\rangle$ - конечная группа диэдра, подгрупша $M=T D$ конечна. По условиям теоремы $M<L<G, L-$ конечная простая группа. Снова применяя лемму 1.6, заключаем, что $L \simeq L_{2}\left(2^{n}\right)$ и $M$ - элементарная абелева 2-группа. Следовательно, все инволюции в $N_{G}(T)$ перестановочны и вместе с единицей составляют нормальную в $N_{G}(T)$ подгруппу, очевидно, совпадающую с силовской 2-подгруппой $U$. Следовательно, подгруппа $\langle U, K\rangle=U \lambda K$ локально конечна и содержит конечную подгруппу $S=V \lambda K,|V|=2^{m}>8$. Как и выше, $S<L<G$, где $L \simeq L_{2}\left(2^{n}\right)$. Однако последнее противоречит лемме 1.9. Полученное противоречие означает, что насьшающее множество $\mathfrak{F}$ не содержит групп, изоморфных $J_{1}$ и $\operatorname{Re}(q)$.

Аналогичными рассуждениями доказывается, что $\mathfrak{F}$ не содержит и групा $L_{2}(q)$, где $q=3,5 \bmod 8$. Следовательно, $\mathfrak{F}$ состоит из групп $L_{2}(q)$, где $q$ - степень 2 . В частности, $C_{G}(i)$ - элементарная абелева 2-группа для любой инволюции $i \in U$ и потому $C_{G}(i)=U$. Последнее означает, что подгруппа $U$ сильно изолирована в $G$, а eе нормализатор $B$ сильно вложен в $G$. Применяя теорему 1 , убеждаемся в справедливости доказьваемой леммы.

На основании лемм 3.2-3.4 далее считаем, что некоторая силовская 2 -подгруппа в $G$ является элементарной абелевой порядка 8.

ЛЕмма 3.5. Все инволюиии в $G$ сопряжены.

ДокАЗАТЕЛЬСтво. Пусть $i, j$ - произвольные инволюции из $G$. В силу периодичности группы $G$ подгруппа $L=\langle i, j\rangle$ конечна и по условиям теоремы $L \leqslant M \in \mathfrak{F}$. По лемме 1.6 $M$ изоморфна одной из групп $J_{1}, L_{2}(4), L_{2}(8), \operatorname{Re}\left(3^{2 n+1}\right), L_{2}(q)$, где $q=3,5$ $\bmod 8$. Ввиду лемм $1.8-1.13$ все инволюции в $M$ сопряжены. Итак, лемма верна.

ЛЕмма 3.6. Пусть $T$ - произвольная силовская 2-подгруппа группы G. Тогда справедливы следующие утверждения:

1. $T$ - әлементарная абелева группа порядка $8, C_{G}(T)=T$ и все силовские 2-подгруппь группь $G$ сопряхсены;

2. $N_{G}(T)=T \lambda(\langle b\rangle \lambda\langle d\rangle)$, әде $\langle b\rangle \lambda\langle d\rangle$ - әруппа Фробениуса порядка 21, $\left|C_{T}(d)\right|=2$ и $T \lambda\langle b\rangle-$ группа Фробениуса порядка 56

3. для любой подгруппь $V<T$ порядка $4 C_{G}(V)=T$.

ДоКАЗАТЕЛЬСТВО. 1. Ввиду условий теоремы и леммы 1.3 все силовские 2-подгруппы в $G$ сопряжены. Поэтому $T$ - элементарная абелева группа порядка 8 . Предположим, что $T \neq C_{G}(T)$ и $b \in C_{G}(T) \backslash T$. В силу периодичности групшы $G$ подгруппа $K=\langle b, T\rangle$ конечна. По условиям теоремы $K \leqslant M \in \mathfrak{F}$. Ввиду леммы 1.6, включения $T \leqslant K$ и леммы 1.8 заключаем, что $M$ изоморфна одной из групш $J_{1}, L_{2}(8), R e\left(3^{2 n+1}\right)$. Однако по леммам 1.9-1.13 $C_{M}(T)=T$. Следовательно, и $C_{G}(T)=T$. Утверждение доказано.

2. Учитывая первое утверждение леммы, заключаем, что $H=N_{G}(T)$ - конечная группа. Как и выше, $H \leqslant M \in \mathfrak{F}$ и $M$ изоморфна одной из групп $J_{1}, L_{2}(8), \operatorname{Re}\left(3^{2 n+1}\right)$. Если хотя бы одна из таких подгрупп $M$ изоморфна $J_{1}$ или $R e\left(3^{2 n+1}\right)$, то утверждение вытекает из лемм $1.11,1.13$. Предположим теперь, что все группы из $\mathfrak{F}$, содержащие подгруппу $H$, изоморфны $L_{2}(8)$. В этом случае $H=T \lambda K$ - групша $K$ - циклическая группа порядка 7 (лемма 1.9). Если множество $\mathfrak{F}$ полностью состоит из подгрупп, изоморфных $L_{2}(8)$ и $L_{2}(4)$, то ввиду леммы $1.9 C_{G}(t)=T$ для любой 
инволюции $t \in T$. Но тогда по лемме $1.5 G$ - почти локально разрешимая группа, вопреки лемме 3.1. Следовательно, $\mathfrak{F}$ содержит также и подгруппы, изоморфные $L_{2}(q)$, где $q=3,5 \bmod 8$. Пусть $M$ - одна из таких подгрупш и $V=T \cap M$ - силовская 2-подгрупша в $M$. По лемме 1.8 подгрупп $N_{M}(V)$ изоморфна знакопеременной группе степени 4 и содержит элемент $b$ порядка 3. Тем же методом, что и в лемме 3.4 , доказьвается, что в $N_{G}(V)$ подгруппа $T$ нормальна и мы можем образовать подгруппу $R=T \lambda\langle b\rangle$. По условиям теоремы $R<L_{2} \in \mathfrak{F}$, противоречие. Таким образом, утверждение 2 справедливо. Легко убедиться, что приведенные рассуждения годятся и для доказательства утверждения 3 леммы. Следовательно, лемма верна.

Введем некоторые обозначения. Пусть $a$ и $z$-различные инволюции из $T, C=C_{G}(a)$, $C_{z}=C_{C}(z)$.

Лемма 3.7. Подәрynna $C_{z}=\langle a\rangle \times\left(B_{z} \lambda\langle t\rangle\right)$, әде $T=\langle a\rangle \times\langle z\rangle \times\langle t\rangle u B_{z}-$ бесконечная локально ииклическая $3^{\prime}$-группа, инвертируемая инволюиией $t$.

ДокАЗАТЕЛЬСТво. Очевидно, что $T=\langle a\rangle \times\langle z\rangle \times\langle t\rangle$, где $t$ - инволюция. В силу леммы $3.6 C_{C_{z}}(t)=T$ и по лемме 1.5 подгрупша $C_{z}$ локально конечна.

Предположим, что $C_{z}$ - конечная подгруппа. Тогда по лемме 1.5 подгруппа $C$ локально конечна и почти разрешима. Пусть $R(C)$ - локально разрешимьй радикал в $C$ и $m=|C: R(C)|$. Выберем в $C$ конечную подгруппу $K$ порядка большего, чем $2 m,\left|J_{1}\right|$, $\left|L_{2}(8)\right|$, содержащую подгруппу $T$. Ввиду условий теоремы $K<M \in \mathfrak{F}$. Учитывая включение $S<M$, порядок $K$ и лемму 1.8 , приходим к вьводу, что $M \simeq \operatorname{Re}\left(3^{2 n+1}\right)$. По лемме $1.11 C \cap M=\langle a\rangle \times L$, где $L \simeq L_{2}\left(3^{2 n+1}\right)$ - простая группа, причем понятно, что $|L|>m$. Но это противоречит равенству $m=|C: R(C)|$. Следовательно, подгруппа $C_{z}$ бесконечна.

Покажем, что любая конечная подгруппа $V$ из $C_{z}$, содержащая подгруппу $T$, имеет вид $V=\langle a\rangle \times D_{V}$, где $D_{V}$ - группа диэдра, не содержащая 3-элементов. Поскольку $C_{z}$ локально конечна, последнее достаточно установить для подгрупा $V<C_{z}$, порядок которых больше, чем $\left|J_{1}\right|,\left|L_{2}(8)\right|$. Пусть $|V|>\left|J_{1}\right|,\left|L_{2}(8)\right|$. По условиям теоремы $V<M \in \mathfrak{F}$. Ввиду включения $T<M$, выбора порядка групшы $V$ и леммы 1.6 имеем $M \simeq \operatorname{Re}\left(3^{2 n+1}\right)$. По лемме $1.11 C \cap M=\langle a\rangle \times L$, где $L \simeq L_{2}\left(3^{2 n+1}\right)$. Ввиду леммы 1.8 для любой инволюции $k \in L$ подгруппа $C_{L}(k)=L_{k}$ диэдральна и не содержит 3 -элементов. Если $z \in L$, то можно считать, что $z=k$ и $D_{V}$, как подгрупа из $L_{k}$, также есть группа диэдра. Если $z \notin L$, то $z=a k$, где $k \in L$. Учитывая, что $L_{k}=(\langle k\rangle \times\langle b\rangle) \lambda\langle t\rangle$, где $b$ - элемент нечетного порядка и $t-$ инволюция из $S$, инвертирующая элемент $b$, заключаем, что подгруппа $D_{1}=(\langle a k\rangle \times\langle b\rangle) \lambda\langle t\rangle$ является группй диэдра без 3-элементов. Следовательно, $D_{V}=D_{1} \cap V$ - группа диэдра и $V=\langle a\rangle \times D_{V}$.

Теперь нетрудно убедиться, что все элементы нечетньх порядков из $C_{z}$ вместе с единищей составляют бесконечную локально циклическую $3^{\prime}$-подгруппу $B_{z}$. При этом каждая инволюция $t \in T$, отличная от инволюций $a, z, a z$, инвертирует подгруппу $B_{z}$. Лемма доказана.

Лемма 3.8. Подгруппа $C$ локально конечна $u C=\langle a\rangle \times L$, где $L \simeq L_{2}(Q), Q-$ локально конечное поле характеристики 3. 
ДокАЗАТЕЛЬСТво. Как локально циклическая группа $B_{z}$ из леммы 3.7 представима в виде объединения $\cup B_{i}$ цепочки

$$
B_{1}<B_{2}<B_{3}<\cdots
$$

конечных циклических подгруп $B_{i}$, причем без ограничения общности можно считать, что $\left|B_{1}\right|>\left|J_{1}\right|,\left|L_{2}(8)\right|$. Образуем подгрупшы $K_{i}=\langle a\rangle \times\left(B_{i} \lambda\langle t\rangle\right)$, где $t \in T \backslash\{1, a, z, a z\}$ (лемма 3.7). По условиям теоремы $K_{i}<M_{i} \in \mathfrak{F}$ и ввиду включения $T<M_{i}$ и леммы 1.6 $M_{i} \simeq \operatorname{Re}\left(3^{2 n_{i}+1}\right)$. Множество $X \subseteq \mathfrak{F}$ подгрупп $M_{i}$ бесконечно и $C_{i}=M_{i} \cap C=\langle a\rangle \times L_{i}$, причем $V_{i}=L_{i} \cap T$ - силовская 2 -подгруппа в $L_{i}$.

Так как подгрупп $V_{i}<S$ конечное число, то $T$ содержит четверную подгрупу $V$, а множество $X$ - бесконечное подмножество $Y$, состоящее из подгрупп $M_{i_{1}}<M_{i_{2}}<\cdots$, для которых $V=V_{i_{1}}=V_{i_{2}}<\cdots$. Нетрудно убедиться, что $\cup_{k} B_{i_{k}}$ совпадает с $B$ и аналогично $\cup_{k}\left(B_{i_{k}} \cap L_{i_{k}}\right)=B$. Поэтому можно считать, что уже $X=Y$ и $B_{i}=B_{z} \cap L_{i}$ для $i=1,2, \ldots$ Кроме того, на основании лемм $3.7,1.11$ считаем, что $V=\langle z\rangle \times\langle t\rangle$ и $C_{L_{i}}(z)=B_{i} \lambda\langle t\rangle$.

По лемме 1.8 инволюции $z$ и $t$ сопряжены в каждой из подгрупп $L_{i}$, в частности, подгруппа $C_{t}=C_{C}(t)$ сопряжена с подгруппой $C_{z}$ и имеет то же строение, т.е. $C_{t}=\langle a\rangle \times$ $\left(B_{t} \lambda\langle z\rangle\right)$, где $B_{t}$ - бесконечная локально циклическая группа, сопряженная с подгруппой $B_{z}$ и инвертируемая инволюцией $z$ (лемма 3.7 ). Как локально циклическая группа $B_{t}$ для каждого $i=1,2, \ldots$ содержит в точности одну подгрупшу $D_{i}$ порядка $\left|B_{i}\right|$. При этом понятно, что подгрупшы $D_{i}$ составляют возрастающую цепочку

$$
D_{1}<D_{2}<D_{3}<\cdots
$$

объединение $\cup D_{i}$ подгрупп которой совпадает с $B_{t}$.

Очевидно, что $C_{L_{i}}(t)=D_{i} \lambda\langle z\rangle$, а по лемме $1.8 L_{i}=\left\langle z, t, B_{i}, D_{i}\right\rangle$. Поэтому из (5) и (6) следует, что подгруппы $L_{i}$ составляют цепочку

$$
L_{1}<L_{2}<L_{3}<\cdots
$$

В силу леммы 1.2 объединение $L=\cup L_{i}$ членов цепочки (7) изоморфно групше $L_{2}(Q)$ над локально конечным полем $Q$ характеристики 3 . При этом подгруппа $R=\langle a\rangle \times L$ содержит подгруппы $C_{z}$ и $C_{t}$.

Предположим, что $R \neq C$. Рассмотрим фактор-групу $\bar{C}=C /\langle a\rangle$. Так как силовская 2-подгрупша в $C$ элементарная абелева, то образы $\bar{C}_{z}$ и $\bar{C}_{t}$ совпадают в $\bar{C}$ с централизаторами инволюций $\bar{z}$ и $\bar{t}$. Понятно также, что $\bar{L} \simeq L$, в $\bar{L}$ все инволюции сопряжены (лемма 1.8 ) и $\bar{C}_{z}, \bar{C}_{t}<\bar{L}$. Отсюда заключаем, что подгрупша $\bar{L}$ сильно вложена в $\bar{C}$ (лемма 1.15).

Пусть $\bar{k}$ - произвольная инволюция из $\bar{C} \backslash \bar{L}$. Из лемм $1.16,1.8$ следует, что $\bar{L}_{\bar{k}}=$ $\bar{L} \cap \bar{L}^{\bar{k}}=\bar{B}$ - подгруппа Бореля в $\bar{L}$. Как вытекает из утверждения 4 леммы 1.8 и утверждения 2 леммы $1.17, \bar{k}$ действует на $\bar{B}$ без неподвижных точек. Ввиду периодичности $\bar{B}$ это равносильно коммутативности группы $\bar{B}$. Но по лемме $1.8 \bar{B}=\bar{P} \lambda \bar{H}-$ группа $\Phi$ робениуса, противоречие. Следовательно, $R=C$ и лемма доказана. 
Лемма 3.9. Группа $G$ локально конечна и изоморфна әруппе $\operatorname{Re}(Q)$, әде $Q$ локально конечное поле характеристики 3.

ДокАЗАТЕЛЬСТво. Воспользуемся рассуждениями из доказательства леммы 3.8. Понятно, что цепочке $(7)$ подгрупп $L_{i} \simeq L_{2}\left(Q_{i}\right)$ соответствует цепочка конечных полей

$$
Q_{1} \subseteq Q_{2} \subseteq Q_{3} \subseteq \cdots
$$

объединение $\cup Q_{i}$ членов которой совпадает с бесконечным локально конечным полем $Q$ характеристики 3 . При этом подгрупшы $L_{i}$ определялись как нормальные дополнения к подгрупше $\langle a\rangle$ в групшах $C_{i}=C \cap M_{i}=\langle a\rangle \times L_{i}$, а $M_{i} \simeq \operatorname{Re}\left(Q_{i}\right)$. Ввиду включений (7) подгрупшы $C_{i}$ составляют цепочку

$$
C_{1} \leqslant C_{2} \leqslant C_{3} \leqslant \cdots,
$$

объединение членов которой совпадает с $C$. Покажем, что конечные подгрупшы $M_{i}$ также составляют цепочку

$$
M_{1} \leqslant M_{2} \leqslant M_{3} \leqslant \cdots
$$

Действительно, по построению все подгруппы $M_{i}$ содержат силовскую 2-подгруппу $T$ группы $G$ и ввиду лемм $1.11,3.6$ - ее нормализатор $N_{G}(T)$. По лемме $1.11 M_{i}=\left\langle N_{G}(S)\right.$, $\left.C_{i}\right\rangle$, а из (9) следуют и включения (10).

Пусть $M=\cup M_{i}$ - объединение членов цепочки (10). При этом понятно, что группа $M$ локально конечна и изоморфна $\operatorname{Re}(Q)$ (лемма 1.2$)$.

Предположим, что $M-$ собственная подгруппа в $G$. Ввиду леммы 1.11 все инволюции в $M$ сопряжены, а из леммы 3.8 следует, что $M$ содержит централизатор $C_{G}(a)$ каждой своей инволюции $a$. По лемме 1.15 подгруппа $M$ сильно вложена в $G$. Пусть $j-$ инволюция из $G \backslash M$ и $D=M \cap M^{j}$. По леммам $1.16,1.17$ подгруппа $D$ не содержит инволюций. Ввиду леммы 1.12 возможны четыре случая, рассмотрим каждьй из них в отдельности.

Пусть $D \leqslant O(B)=U \lambda A_{0}$, где $B$ - подгруппа Бореля групшы $M$. Поскольку циклическая подгруппа $H \leqslant B$ содержит инволюцию, подгруппа $B$ не действует сопряжениями транзитивно на множестве всех инволюций из $M$ вопреки леммам $1.16,1.17$. Следовательно, $D \notin O(B)=U \lambda A_{0}$.

Рассмотрим возможности $D \leqslant A_{i} \lambda\left\langle b_{i}\right\rangle$, где $i=1,2,3$ (лемма 1.12). По лемме 1.12 $C_{G}\left(A_{1}\right)$ содержит инволюцию и поэтому $A_{1} \lambda\left\langle b_{1}\right\rangle$ не может действовать сопряжениями транзитивно на множестве всех инволюций из $M$. Значит, $i \neq 1$. А при $i=2,3$ в $M$ найдется инволюция, инвертирующая $A_{i}$ и перестановочная с $b_{i}$. Поэтому и для этих подгрупп $A_{i} \lambda\left\langle b_{i}\right\rangle$ невозможно транзитивное действие на множестве всех инволюций из $M$. Следовательно, $D \notin A_{i} \lambda\left\langle b_{i}\right\rangle, i=2,3$. Последнее означает, что $G=M$ и лемма доказана.

Лемма 3.9 завершает доказательство теоремы 2. 


\section{СПИСОК ЦИТИРОВАННОЙ ЛИТЕРАТУРЫ}

[1] Шлепкин А. К. Сопряженно бипримитивно конечные группы, содержащие конечные неразрешимые подгруппы // Третья международная конференция по алгебре (23-28 августа 1993). Сб. тезисов. Красноярск, 1993. С. 369.

[2] Созутов А. И., Шлепкин А. К. О группах с сильо вложенной подгруппой, насыщенных конечными простыми подгруппами // Международ. алгебраич. семинар, посвященный 70-летию каф. высшей алгебры МГУ (10-12 февраля 1999г.). Тез. докл. М.: МГУ, 1999. С. 53.

[3] Шлепкин А. К. О некоторых периодических группах, насыщенных конечными простыми подгруппами // Матем. труды. 1998. Т. 1. №1. С. 129-138.

[4] Шлепкин А.К., Васильева О.В. О периодических группах с абелевой силовской 2 -подгруппой порядка 8, насьщенных конечньми простьми подгруппами // Вестник КрасГАУ (сдана в печать)..

[5] Созутов А. И. О некоторых бесконечных группах с сильно вложенной подгруппой // Алгебра и логика. 2000. Т. 39. № 5. С. 602-617.

[6] Созутов А. И. Два признака непростоты группы с сильно вложенной подгруппой и конечной инволюцией // Матем. заметки. 2001. Т. 69. № 3. С. 443-453.

[7] Беляев В.В. Локально конечные группы Шевалле // Исследования по теории групп. Свердловск: УНЦ АН СССР, 1984. С. 39-50.

[8] Шунков В.П. О некотором обобщении теоремы Фробениуса на периодические группы // Алгебра и логика. 1967. Т. 6. №3. С. 113-124; О теории периодических групп // Докл. AH CCCP. 1967. T. 175. №6. C. 1236-1237.

[9] Шунков В.П. Об одном классе p-групп // Алгебра и логика. 1970. Т. 9. № 4. С. 484-496.

[10] Беляев В. В. Группы с почти регулярной инволюцией // Алгебра и логика. 1987. Т. 26. № 5. C. 531-535.

[11] Шунков В.П. О периодических группах с почти регулярной инволюцией // Алгебра и логика. 1972. Т. 11. № 4. С. 470-494.

[12] Горенстейн Д. Конечные простые группы. М.: Мир, 1985.

[13] Мазуров В. Д. Конечные группы // Итоги науки и техники. Алгебра. Топология. Геометрия. 1976. Т. 14. С. 5-56.

[14] Бусаркин В. М., Горчаков Ю. М. Конечные расщепляемые группы. М.: Наука, 1968.

[15] Suzuki M. On a class of doubly transitive groups. I // Ann. Math. 1962. V. 75. № 1. P. 105-145; II // Ann. Math. 1964. V. 79. № 3. P. 514-589.

[16] Нужин Я.Н. О строении групп лиева типа ранга 1 // Матем. заметки. 1984. Т. 36. № 2. C. $149-150$.

[17] Левчук В. М., Нужин Я.Н. О строении групп Ри // Алгебра и логика. 1985. Т. 24. № 1. C. $26-41$.

[18] Сыскин С. А. Абстрактные свойства простых спорадических групп // УМН. 1980. Т. 35. № 5. C. 181-212.

[19] Шунков В. П. Мp-группы. М.: Наука, 1990.

[20] Шунков В. П. О вложении примарных элементов в группе. Новосибирск: ВО Наука, 1992.

[21] Измайлов А. Н. Характеризация групп $S L_{2}(K)$ и $S z(K)$ над локально конечным полем характеристики 2 // Алгебра и логика. 1985. Т. 24. № 2. С. 127-172.

[22] Курош А. Г. Теория групп. М.: Наука, 1967.

[23] Хухро Е.И. Нильпотентные группы и их автоморфизмы простого порядка. Фрайбург, 1992.

[24] Холл М. Теория групп. М.: ИЛ, 1962.

[25] Каргаполов М.И., Мерзляков Ю. И. Основы теории групп. М.: Наука, 1977.

[26] Созутов А. И., Сучков Н. М. О бесконечных группах с заданной сильно изолированной 2-подгруппой // Матем. заметки. 2000. Т. 68. № 2. С. 272-285.

(А. И. Созутов) Красноярская государственная

Поступило архитектурно-строительная академия

29.01.2001

(А. К. Шлепкин) Красноярский государственный аграрный университет 